\title{
INDEPENDENCIA Y ECOLOGÍA ECTOMICORRÍCICA EN VARIAS ESPECIES De los GÉneros Quercus, PINUS Y EUCALYPTUS
}

\author{
Juan Andrés ORIA DE RUEDA SALGUERO
}

\begin{abstract}
RESUMEN: Se estudia la micorrización de varias especies de Quercus y Eucalyptus con hongos micorrícicos de los géneros Pisolithus, Krombholziella y Suillus. Se define el Período de Independencia Micorrícica, muy interesante en viveros forestales y de reproducción vegetal. Este período varía según especies y ecología y es mayor con semillas voluminosas que en las de semillas pequeñas.
\end{abstract}

Palabras clave: Independencia Micorrícica, viveros forestales, inoculación.

SUMMARY: Mycorrization of some species of Quercus, Pinus and Eucalyptus with some mycorrhizal fungi: Pisolithus, Krombholziella and Suillus is studied. Micorrhizal Independency Period is defined and results very interesting in forest nursery and tree farms. This period is different according to species and ecology and is larger in big seed species than in small seed species.

Key words: Mycorrhizal Independency, forest nursery, inoculation.

\section{INTRODUCCIÓN}

Con cierta frecuencia se encuentran resultados conflictivos al emplear especies leñosas micorrícicas en restauración del medio natural (Marx, 1980). Sobre todo en condiciones ecológicas difíciles, se hace necesario conocer los requerimientos, ya sean minerales o biológicos, de la simbiosis micorrícicas (Meyer, 1987; Denny \& Wilkins, 1987).

Debido a la diferente especificidad de los inóculos empleados respecto a la planta huésped y, sobre todo, al distinto metabolismo de las especies, variedades y razas, los resultados prácticos, desde un punto de vista técnico, de las restauraciones forestales son a veces contradictorios (Schenck, 1987). 
Existen razas fisiológicas de hongos capaces de asociarse simbióticamente con unos géneros o especies diferentes (Marx, 1982).

Incluso en área reducidas, como es el caso del S.E. de España, concretamente en la provincia de Almería, coexisten numerosas formas biológicas y razas fisiológicas de hongos ectomicorrícicos, como Pisolithus tinctorius (Pers.) Coker \& Couch (Oria de Rueda, 1988, a, b).

Desde el punto de vista técnico resulta fundamental saber el momento óptimo de inoculación, con el fin de mejorar la supervivencia de las plántulas en el lugar definitivo de la plantación o siembra y, consiguientemente, desde el punto de vista económico. Si bien existen firmas que elaboran inóculo comercial, aunque de forma variable (Froidevaux, 1985) los resultados del inóculo comercial son desiguales según los géneros y especies de las plantas inoculadas.

En las restauraciones hidrológicas forestales que se están realizando en el S.E. de España, se viene haciendo necesario el optimizar los procesos de plantación (Simon, 1982).

Desde 1986 venimos estudiando en la provincia de Almería la distribución y utilidad de los hongos ectomicorrícicos (Oria de Rueda, 1987; Calonge \& Oria de Rueda, 1988; Oria de Rueda, 1988).

\section{PROBLEMATICA DE LA INDEPENDENCIA MICORRÍCICA}

Venimos observando el hecho de que las especies del género Quercus se comportan de una manera contradictoria al ser inoculadas en sus primeros meses. Se llega al punto de que las plántulas micorrizadas de Quercus ilex L. y Quercus coccifera L. muestran igual o inferior crecimiento, probando que son independientes, en general, de la micorrización, durante un cierto timpo. Las plantas de gran tamaño, se comportan de diferente manera que aquellas especies de plantas que utilizan las sustancias nutritivas de la semilla sólo durante la germinación y seguidamente dependen de la fotosíntesis para obtener fotosintato. En el caso especial de Quercus coccifera $\mathrm{L}$., ésta es capaz de seguir utilizando los nutrientes de la semilla hasta finales del segundo año de vida, agotándolos casi completamente.

Asimismo, las especies del género Quercus emplean, en su primera edad, los nutrientes sobre todo en profundizar y aumentar el sistema radical, a expensas del crecimiento del tallo y de las hojas. Hemos medido raícex pivotantes de Quercus ilex L. de un año que profundizaban $90 \mathrm{~cm}$, mientras que la altura del tallo desde el cuello de la raíz no llegaba a $8 \mathrm{~cm}$.

Como los hongos ectomicorrícicos producen proliferación de raíces cortas y ramificación de las mismas, mientras que los Quercus presentan naturalmente en sus primeros años una raíz fuerte pivotante que profundice, con escasez de raíces secundarias, se plantea un conflicto: a la plántula «no le interesa» que las raíces sean cortas y ramificadas, en contra de su principal objetivo. 
Hemos comprobado que durante los primeros 10-14 meses de vida de las plántulas de Quercus, éstas se mantienen totalmente independientes de la inoculación, presentando muy escasas, e incluso inexistentes, micorrizas. En el caso de Quercus coccifera cultivado en tierra de $\mathrm{pH}$ elevado de $\mathrm{pH}\left(\mathrm{H}_{2} \mathrm{O}\right)=8,4$, obervamos que la independencia llegaba al tercer año, pese a que no quedaban reservas en los cotiledones de las bellotas, pero pensamos que sí existían en las profundas raíces que desarrollaban en los primeros 8 meses de vida las plantas.

Uno de los aspectos importantes encontrados al estudiar la micorrización de Quercus robur L. al ser inoculados con Krombholziella nigrescens (Rich \& Roze) Sut., es que las plántulas de 2 años (procedentes de semilla recogida en la provincia de La Rioja, en el límite de su área) testigo no micorrizadas, presentan una relación diámetro de la raíz/diámetro del tallo igual a 2,9 y al ser micorrizadas en sus primeros 10 meses presentan una relación igual a 2,2, es decir, la micorrización produce un cambio en la relación raíz/tallo, como consecuencia del aumento de la producción de la parte aérea de la planta frente a la subterránea.

\section{PERÍODO DE INDEPENDENCIA MICORRÍCICA}

Definimos el Período de Independencia Micorrícica como el intervalo de tiempo en el que la plántula, desde la germinación de la semilla, puede vivir y crecer correctamente sin necesitar la asociación simbiótica micorrícica. Las sustancias nutritivas que contiene la semilla pueden ser utilizadas, en el caso especies arbóreas forestales, sin la colaboración de los hongos micorrizógenos. El PIM varía según nuestras investigaciones, no sólo de especie a especie sino también debido a la procedencia y origen de las semillas, forma y densidad de las mismas, características del medio de germinación y lugar de crecimiento, etc.

Para especies del género Quercus el PIM varía desde los 10 a los 30 meses. En Eucalyptus brockwavi, Gard. especie empleada en zonas áridas en Almería y región norsahariana por los servicios forestales, el PIM se sitúa en torno a los 6 meses mientras en Pinus halepensis Miller en los 7 a 8 meses. Si se separan los cotiledones de la semilla, durante la germinación, el PIM se acaba bruscamente. Creemos que es del mayor interés conocer el Período de Independencia Micorrícica, para averiguar cuando es el momento óptimo de la micorrización controlada, con hongos ectomicorrizógenos en los viveros forestales.

\section{MÉTODO Y TRABAJO EXPERIMENTAL}

Se recogieron semillas de Quercus ilex, L.Quercus coccifera, L.Pinus halepensis Miller y Eucalyptus brockwayii Gard. en la provincia de Almería. Asimismo se recogieron bellotas de Quercus robur L. en las provincias de La Rioja y Madrid. Se 
realizaron inoculaciones, por medio de esporas o por medio de micelio obtenido por cultivo según métodos clásicos (Marx, 1969, Norkrans, 1949, Oria de Rueda, 1988a).

Los carpóforos de Pisolithus tinctorius (Pers.) Coker \& Couch y Scleroderma verrucosum (Bull: Pers.) Grev utilizados, fueron recogidos en la provincia de Almería, los de Krombholziella nigrescens (Rich \& Roz) Sut. en Ezcaray (La Rioja) y las de Krombhoziella corsica Bouch. en la Casa de Campo (Madrid).

Los carpóforos de Suillus bellinii (Inzen.) Watl. fueron recogidos en la Sierra de Lúcar (Almería), mientras los de Suillus collinitus (Fr.) Kuntze lo fueron en Cuevas de Almanzora, también en la misma provincia. Los resultados de este trabajo se observan en las gráficas que se exponen a continuación. En los ejes de ordenadas aparecen las alturas de las plántulas en centímetros mientras en los ejes de abscisas aparecen los distintos tratamientos.

\section{RESULTADOS}

\section{APLICACIONES DE LAS ECTOMICORRIZAS DE ZONAS ÁRIDAS.}

Las micorrizas de zonas áridas y desérticas poseen un gran valor en plantaciones y restauración forestal (Miller, 1979; Mejstric \& al., 1983).

Si bien las micorrizas VA predominan en estas regiones secas (Read, 1986), también es posible encontrar ectomicorrizas en abundancia (Oria de Rueda, 1987).

La mayor absorción de agua por parte del micelio extramatricial y la mayor capacidad de traslocación del fórforo, son dos ventajas suficientemente comprobadas en las micorrizas en zonas secas (Read, 1986).

El mantenimiento de una amplia red externa de micelio extramatricial es de gran importancia en zonas áridas y semiáridas, como ocurre, por ejemplo, en el caso de Pisolithus tinctorius (especie que vamos a estudiar más detenidamente) cuyo empleo ha tenido éxito en regiones afectadas por fuertes sequías (Schram, 1966).

En la provincia de Almería, y en S.E. de España en general, la erosión laminar y, sobre todo, la erosión en regueros y barrancos es considerable, principalmente en las comarcas en las que predominan margas poco consolidadas.

En varias de estas zonas las arcillas tienen un comportamiento dispersivo que impide a la vegetación ocupar el suelo debido a un proceso de hinchamiento/ agrietamiento y desmoronamiento de las mismas. Según nuestras observaciones las abundantes hifas extramatriciales invaden de tal manera el horizonte superior del suelo ( $10 \mathrm{~cm}$ de profundidad) que el proceso de dispersión, característico de las zonas «bad-land»se atenúa considerablemente.

Esta ventaja de la agregación de las partículas del suelo se observa más claramente en los terrenos sueltos, de aluviones o costeros, con ventajas claras frente a la erosión.

De igual manera, la producción de raíces cortas que son estimuladas por los hongos micorrizógenos, representa una utilidad en la lucha contra la dispersión y desmoronamiento de terrenos poco consolidados. 

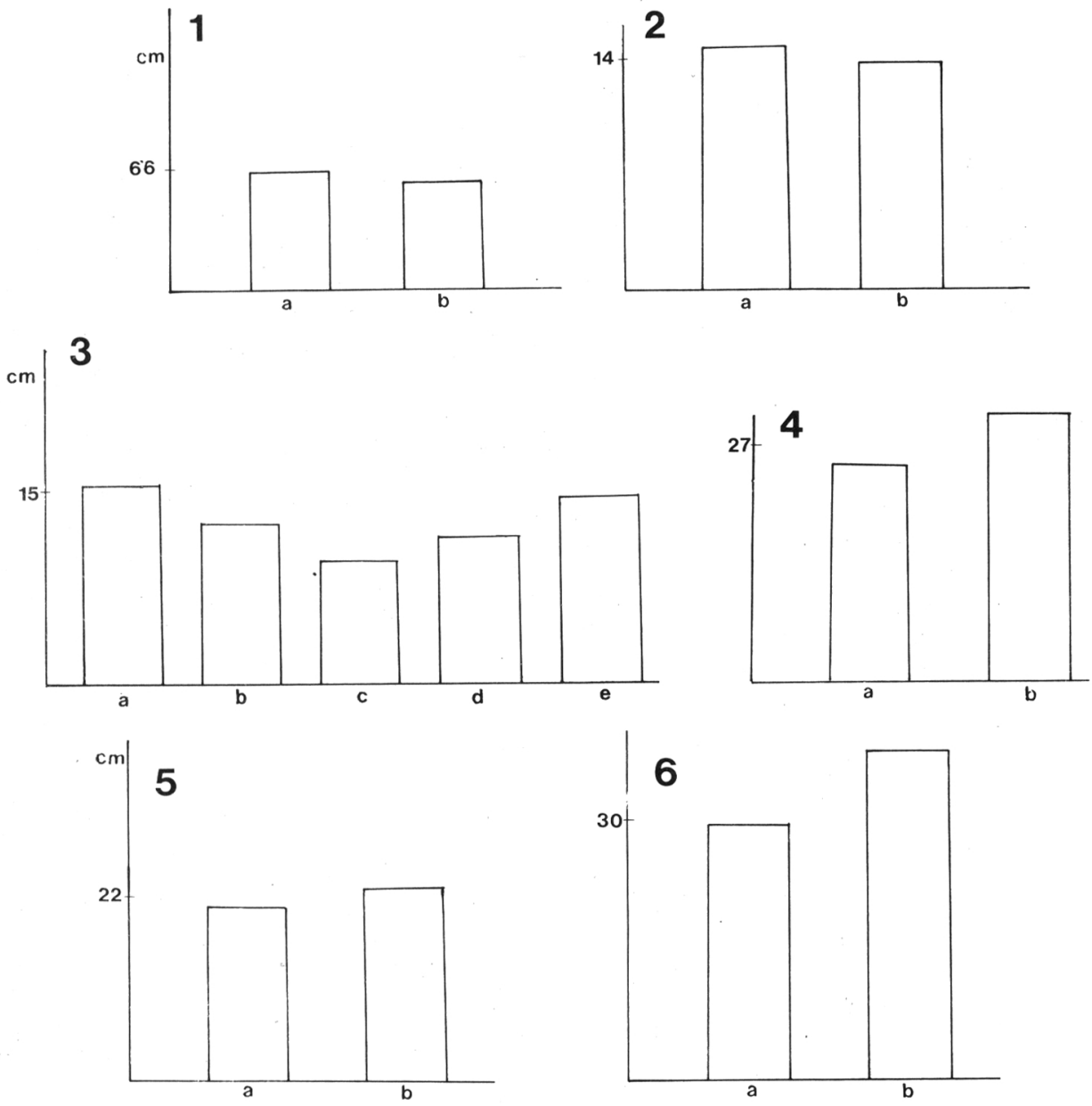

Figs. 1-6. 1: Altura de las plántulas de Quercus coccifera, de diez meses de edad. a) control, no micorrizado. b) inoculadas con Pisolithus tictorius. 2: Altura en $\mathrm{cm}$ de las plántulas de Quercus coccifera de 36 meses de edad igual al período de independencia micorrícica para semillas recogidas en la Sierra de Oria (Almería) y sustrato edáfico fuertemente básico. a) control, no inoculada. b) inoculada con Pisolithus tinctorius. 3: Altura en cm de las plántulas de Quercus ilex de catorce meses de edad. a) No micorrizada (control). Altura de la planta $15 \mathrm{~cm}$. Diámetro del cuello de la raíz, $3,3 \mathrm{~mm}$. b) Micorrizada con esporas de $P$. tinctorius procedente de Vélez Blanco. Altura $12 \mathrm{~cm}$. Diámetro del cuello de la raíz $2,75 \mathrm{~mm}$. c) Micorrizada con tierra de bosque. Altura $10 \mathrm{~cm}$. Diámetro del cuello de la raíz, 2,4 mm. d) Inoculada con esporas de $P$. tinctorius del eucaliptal de Cuevas de Almanzora. No se micorrizó. Diámetro 3,4 mm. e) Inoculada y micorrizada con esporas de $P$. tinctorius procedente de Madrid (Casa de Campo). Diámetro del cuello de la raíz 3,1 mm. 4: Altura en cm de las plántulas de Quercus ilex de 36 meses de edad (PIM igual a 24 meses). a) Control, no micorrizado. b) Inoculado con Krombholziella corsica. 5: Altura en cm de las plántulas de Quercus robur de ocho meses de edad. a) Inoculado con Krombholziella nigrescens. b) Control, no micorrizado. 6: Altura en cm de las plántulas de Quercus robur de 24 meses de edad. a) Control, no micorrizado. b) Micorrizado con Krombolziella nigrescens. Período de Independencia Micorrícica estimado en quince meses. 
Otro aspecto a destacar en terrenos con dominio de marga y pizarras, en los que hemos recogido ejemplares de Pisolithus tinctorius y Scleroderma verrucosum (Bull: Pers.) Grev (Calonge \& Oria de Rueda, 1988) es que el micelio ex tramatricial de estos hongos micorrizógenos tienen mucho menor diámetro que la raíz más fina del árbol, unas 1000 veces menos ( $1 \mathrm{~mm}$ frente a $1 \mu \mathrm{m}$ ), por lo que las hifas pueden introducirse entre las láminas de las pizarras y margas, donde las raíces no tienen acceso por imposibilidad física de atravesar estas rocas sedimentarias. Se esta manera, el micelio extramatricial es capaz de explorar zonas inaccesibles para las raíces.
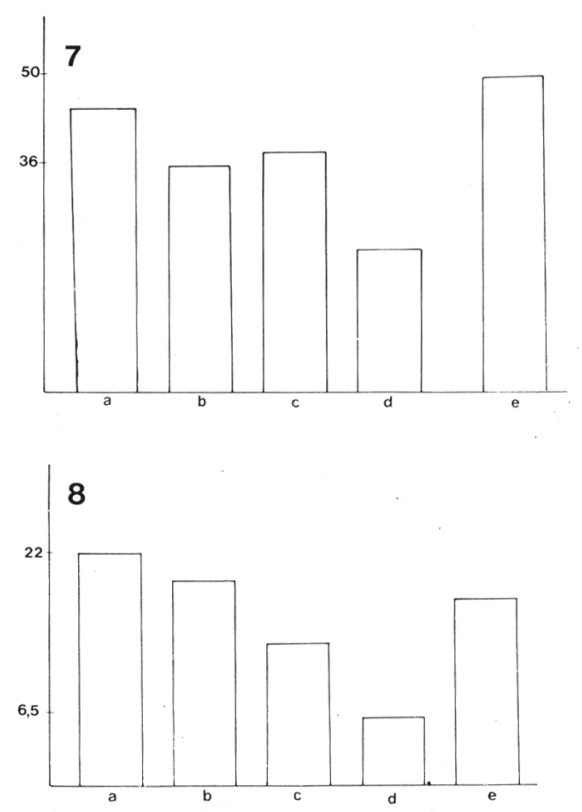

Figs. 7-8. 7: Altura en cm de las plántulas de Eucalyptus brockwayi de catorce meses de edad. a) Micorrizado con esporas de P. tinctorius recogidas en Cuevas de Almanzora. Altura de la planta: $45 \mathrm{~cm}$. Diámetro del cuello de la raíz, 3,9 mm. b) Control. Sin micorrizar. Diámetro 2,6 mm. c) Inoculado con esporas de P. tinctorius recogidas en el encinar de la Casa de Campo (Madrid). No se produjeron ectomicorrizas. Diámetro 3,1 mm. d) Inoculado con tierra recogida en un saladar de Cuevas de Almanzora. No se formaron ectomicorrizas. Sólo micorrizas VA. Diámetro, $2,1 \mathrm{~mm}$. e) Inoculado y micorrizado con raíces de Eucalyptus occidentalis, recogidas en Cuevas de Almanzora. Diam. 4,2 mm. Alt. $51 \mathrm{~cm}$. En las raíces de estas plántulas se formaron abundantes ecto y endomicorrizas. 8: Altura en $\mathrm{cm}$ de las plántulas de Pinus halepensis de diez meses de edad. (PIM = 8 meses). a) Micorrizado con Suillus collinitus. Altura de la planta 22,3 cm. Diámetro del cuello de la raíz 3,6 mm. b) Micorrizado con Suillus bellinii. Altura: $19,5 \mathrm{~cm}$. Diámetro del cuello de la raíz: $3,8 \mathrm{~mm}$. c) Inoculado con $P$. tinctorius f. tinctorius procedente del eucaliptal de Cuevas. No micorrizó. Altura: $14 \mathrm{~cm}$. Diámetro del cuello de la raíz: $2,1 \mathrm{~mm}$. d) Control No micorrizado. Diámetro: 2,6 mm. e) Micorrizado con tierras de bosque procedente del pinar de Pinus halepensis de Bayarque (Filabres). Diámetro: $3,1 \mathrm{~mm}$. 
En muchas de estas margas y pizarras se infiltra agua de luvia y se acumula una cierta humedad que es aprovechada por las hifas y, por lo tanto, por el árbol huésped. Después de atravesar verdaderas rocas gracias a esto, cuando se acercan a la superficie se producen con profusión carpóforos que salen literalmente de la roca. Conservamos varios de ellos que han salido en rocas a varios metros de los árboles huésped. De esta manera hay eucaliptos creciendo en zonas de pizarra pura en la comarca de Gérgal.

Por otra parte, en los suelos clasificados como Aridisoles con costras petrocálcicas situadas a menos de $1 \mathrm{~m}$ de profundidad, (Paleorthids) y que son muy frecuentes en la zona costera y media de la provincia y, en general, todos los suelos con costras endurecidas en la superficie, los hongos ectomicorrícicos tienen una actuación directa.

Cuando emergen los carpóforos, desde zonas cercanas a las micorrizas, levantan las costras y piedras que encuentran a su paso.

Esto ha sido observado en el caso de Suillus collinitus en Almería (Oria de Rueda, 1987); pero donde se ve de manera más clara y contundente es en el caso de Pisolithus tinctorius. Algunos de los carpóforos recogidos en la provincia, superan los 700 grs de peso y $20 \mathrm{~cm}$ de diámetro y son capaces de levantar firmes asfálticos de carreteras y costras petrocálcicas de varios centímetros de grosor.

La ruptura de costras petrocálcicas constituye un objetivo en la agricultura de las zonas áridas (Buol, 1983) y es realizado por los carpóforos de P.tinctorius de manera eficaz; además de la profusión con que salen sus carpóforos después de las lluvias otoñales.

El caso de la ruptura de firmes de las carreteras contituye, en cambio, un grave problema que vamos a analizar detenidamente.

Las raíces micorrizadas tienden a desarrollarse más en ciertas zonas donde hay alguna humedad, como ocurre debajo de piedras planas, que evitan la evaporación del agua del suelo.

En muchas carreteras suele acumularse agua justamente por debajo, debido a la infiltración del agua desde las cunetas y zonas próximas. Si la carretera tiene firme asfáltico impermeable no se evapora el agua infiltrada quedando acumulada. Las raíces de los árboles, sobre todo de los más ávidos de humedad, se desarrollan pronto allí y con ellas las micorrizas. Ocurre entonces que al emerger los carpóforos éstos son capcaces de romper el firme y originar baches de gran diámetro.

Esto ha sido comprobado en California con Pisolithus tinctorius (Burk \& al. 1979), la misma especie que hamos visto rompiendo el firme de la carreteras de Almería. En la carretera de Almería-Cabo de Gata, en otoño de 1987 emergieron del firme asfáltico, sobre todo en los bordes de la carretera, innumerables carpóforos de $P$. tinctorius. Una vez que el carpóforo ha roto el firme, varias especies de plantas como Cynodon dactylon (L.) Pers. son capaces de crecer en las grietas y agujeros colaborando en la destrucción del firme.

La solución a este problema está, ante todo, en no prodigar la plantación de Eucalyptus en los bordes de las carreteras, puesto que, por su elevado crecimiento, 
avidez por el agua y desarrollo superficial de sus raíces, puede producir desperfectos en las vías de transporte.

En otro orden de cosas hay que evitar que debajo de la capa de rodadura (impermeabilizada normalmente por mezclas asfálticas) se acumule humedad, por lo que habrá que tener en cuenta la construcción de la capa de base y subbase según muestra la figura siguiente.

firme

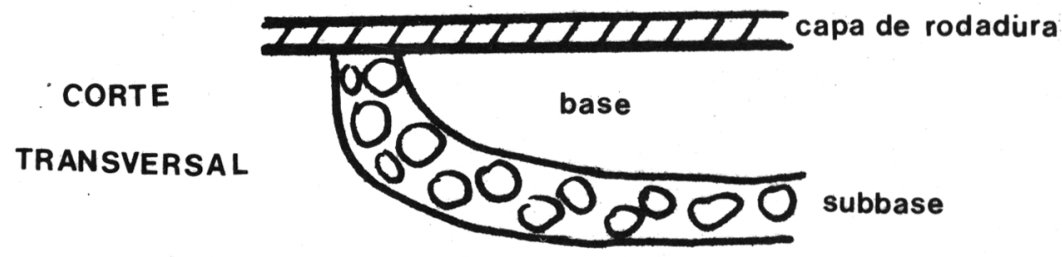

La capa subbase debe fabricarse con material lo más grueso posible, que impida que el agua llegue por capilaridad a la capa base (ICONA, 1983).

\section{AGRADECIMIENTOS}

Queremos expresar nuestro agradecimiento a los ingenieros del IARA, D. José Antonio de Simón y D. Estanislao de Simón, así como al personal del Servicio Forestal de la Confederación Hidrográfica del Sur de Almería y del IARA de Almería.

Nuestro reconocimiento, igualmente, al catedrático de ETSI de Montes (Unidad de Botánica), D. J. Ruiz de la Torre por sus ayudas de todo tipo en la realización de este trabajo. Finalmente agradecemos a A. Ibañez los medios de cultivo.

\section{BIBLIOGRAFÍA}

BUOL, S.W., F.D. HOLE \& R.J. McCRACKEN -1983- Génesis y clasificación de suelos. México.

BURK, W.R. \& G.F. LUPONE -1979- Pisolithus tinctorius, a pavement breaker in Southern California. Mycotaxon, 8(2):469-470.

CALONGE, F.D. \& J.A. ORIA DE RUEDA -1988- Aportación a la micoflora de la provincia de Almería. Bol. Soc. Micol. Madrid, 12:93-106.

DENIS, H.J. \& D.A. WILKINS -1987- Zinc tolerance in Betula spp. New Phytologist, 106(3):545-553.

FROIDEVAUX, L. -1985- Sélection d'ectomycorrhizes spécifiques pour le reboisement des sols toxiques ou dégradés. Jour. Forest. Suisse, 136(6):445-456.

HARLEY, J.L. -1969- The biology of mycorrhiza. Plant. Sci. Monogr. 2nd. Hill. London.

HARLEY, J.L. \& S.E. SMITH -1983-Mycorrhizal simbiosis. Academic. Press New York.

HONRUBIA, M. -1982- Aportación al conocimiento de los hongos del S.E. de España. Tesis doctoral. Universidad de Murcia. 
ICONA -1983-Planificación y proyecto de vias forestales. Madrid.

MARX, D.H. -1969- Phytopathology, 59:153-163.

MARX, D.H. -1980- Ectomycorrhizal fungus inoculation: a tool for improving forestation practices. In Tropical Mycorrhiza Research: 13-71. Oxford Univ. Press.

MEJSTRIK, V.K. \& P. CUDLIN -1983- Mycorrhiza in some plant desert species in Algeria. In Plant and Soil, 71:363-366.

MEYER, F.H. -1987- Extreme Standorte und Ektomykorrhiza. Angewandte Botanik, 61(19):39-46.

MILLER, R.M. -1979- Some occurrence of vesicular arbuscular mycorrhizain natural and disturbed ecosystems of the Red Desert. Can. J. Bot., 57:619-633.

NORKRANS, B. -1949- Svensk. Bot. Tidskr., 43:485-490.

ORIA DE RUEDA, J.A. -1987-Ecología del género Suillus en Almería y aplicación de las micorrizas de zonas áridas en repoblaciones forestales. Univ. Politécnica. Madrid.

ORIA DE RUEDA, J.A. -1988-Ecología de Pisolithus tinctorius en la provincia de Almería (S.E. de España) IV Reunión Conjunta de Micología. (inéd.).

READ, D.J. -1986- Non nutricional effects of mycorrhizal infection. in Mycorrhizae: physiology and genetics. INRA. París.

SCHRAMM, J.R. -1966- Trans. Am. Phil. Soc., 56:1194.

SIMÓN, DE E. -1982- Repoblaciones forestales en regiones semiáridas. Instituto de estudios almerienses. Almería.

(Aceptado para su publicación en Junio de 1.990 )

Dirección del autor: Departamento de Silvopascicultura (Unidad de Botánica). ETSI de Montes. Ciudad Universitaria. 28040. Madrid. 
